\title{
PLAG1, SOX10, and Myb Expression in Benign and Malignant Salivary Gland Neoplasms
}

\author{
Ji Hyun Lee · Hye Ju Kang \\ Chong Woo Yoo - Weon Seo Park \\ Junsun Ryu ${ }^{1}$ - Yuh-Seog Jung ${ }^{1}$ \\ Sung Weon Choi $^{2} \cdot$ Joo Yong Park ${ }^{2}$ \\ Nayoung Han
}

Department of Pathology, National Cancer Center, Goyang; ${ }^{1}$ Head and Neck Oncology Clinic, National Cancer Center, Goyang; ${ }^{2}$ Oral Oncology Clinic, National Cancer Center, Goyang, Korea

Received: July 24, 2018

Revised: October 10, 2018

Accepted: October 11, 2018

\section{Corresponding Author}

Nayoung Han, MD, PhD

Department of Pathology, National Cancer Center, 323 Ilsan-ro, Ilsandong-gu, Goyang 10408, Korea Tel: +82-31-920-1756

Fax: +82-31-920-1369

E-mail: hanny@ncc.re.kr

\begin{abstract}
Background: Recent findings in molecular pathology suggest that genetic translocation and/or overexpression of oncoproteins is important in salivary gland tumorigenesis and diagnosis. We investigated PLAG1, SOX10, and Myb protein expression in various salivary gland neoplasm tissues. Methods: A total of 113 cases of surgically resected salivary gland neoplasms at the National Cancer Center from January 2007 to March 2017 were identified. Immunohistochemical staining of PLAG1, SOX10, and Myb in tissue samples was performed using tissue microarrays. Results: Among the 113 cases, 82 (72.6\%) were benign and 31 (27.4\%) were malignant. PLAG1 showed nuclear staining and normal parotid gland was not stained. Among 48 cases of pleomorphic adenoma, 29 (60.4\%) were positive for PLAG1. All other benign and malignant salivary gland neoplasms were PLAG1-negative. SOX10 showed nuclear staining. In normal salivary gland tissues SOX10 was expressed in cells of acinus and intercalated ducts. In benign tumors, SOX10 expression was observed in all pleomorphic adenoma (48/48), and basal cell adenoma (3/3), but not in other benign tumors. SOX10 positivity was observed in nine of $31(29.0 \%)$ malignant tumors. Myb showed nuclear staining but was not detected in normal parotid glands. Four of $31(12.9 \%)$ malignant tumors showed Myb positivity: three adenoid cystic carcinomas (AdCC) and one myoepithelial carcinoma with focal AdCC-like histology. Conclusions: PLAG1 expression is specific to pleomorphic adenoma. SOX10 expression is helpful to rule out excretory duct origin tumor, but its diagnostic value is relatively low. Myb is useful for diagnosing AdCC when histology is unclear in the surgical specimen.
\end{abstract}

Key Words: Salivary gland neoplasms; Immunohistochemistry; Pleomorphic adenoma gene-1; SOX transcription factors; Oncogene proteins v-Myb
Salivary gland tumors are heterogeneous tumors with many variable features including histological morphology, lineage, and biological characteristics. ${ }^{1}$ Recently, specific fusions and overexpression of oncoproteins have been reported in some salivary gland tumors. ${ }^{2}$

Pleomorphic adenoma gene 1 (PLAG1) functions as an oncogene in several human tumors. ${ }^{3}$ Overexpression of PLAG1 promotes mitogenesis, proliferation, apoptosis, and insulin-like growth factor 2 upregulation. ${ }^{4}$

SRY-related HMG-box 10 (SOX10) is known to play a crucial role in the differentiation, maturation, and maintenance of Schwann cells and melanocytes. ${ }^{5}$ SOX10 is a potential marker for acinar and intercalated duct differentiation in salivary gland tumors. ${ }^{6}$

$v-M y b$ is an oncogene homolog of the avian myeloblastosis virus. It acts as a DNA-binding transcription regulator and is a proto-oncogene that plays an important role in controlling prolif- eration and differentiation. ${ }^{7}$

In this study, we investigated PLAG1, SOX10, and Myb protein expression in human salivary gland neoplasm samples and evaluated the association between the expression profiles and histological and clinical features.

\section{MATERIALS AND METHODS}

\section{Patients}

The files of 113 patients with surgically resected primary salivary gland neoplasm who underwent operation at the National Cancer Center from January 2007 to March 2017 were obtained. Three adenoid cystic carcinoma cases reported between April 2017 and June 2018 were additionally obtained. Patient age, sex, histologic type, size, and involved site were evaluated by reviewing medical charts and pathological records. Histologic diagnosis of one case (No. 72) was discussed and revised from adenocarci- 
noma, not otherwise specified (NOS) to adenoid cystic carcinoma. This study protocol was reviewed and approved by the Institutional Review Board (IRB) of the National Cancer Center (NCC2018-0182). The need for informed consent was waived by the IRB.

\section{Tissue microarray}

Tissue array blocks were prepared as described previously. ${ }^{8}$ Suitable areas for tissue retrieval from triplicate 2-mm-diameter cores obtained from the tumor block were marked on standard hematoxylin and eosin-stained sections, punched out from the paraffin block, and inserted into a recipient block. Sections (4- $\mu \mathrm{m}$-thick) were cut from each tissue array block, deparaffinized, and dehydrated. An adequate case was defined as tumor occupying more than $10 \%$ of the core area.

\section{Immunohistochemistry}

Immunohistochemistry (IHC) staining was performed using an automatic immunostainer (Ventana, Tucson, AZ, USA) according to the manufacturer's instructions. The primary antibodies used were as follows: mouse monoclonal anti-PLAG1 (clone 3B7, Novus, Littleton, CO, USA), rabbit monoclonal anti-SOX10 (clone EP268, Bio SB, Santa Barbara, CA, USA), and rabbit monoclonal anti-v-Myb+c-Myb (ab45150, Abcam, Cambridge, UK). The stroma surrounding the tumor cells preserved the normal staining pattern, thereby serving as the internal control. A case was considered positive if any of the cores were stained with any intensity.

\section{Statistical analysis}

The chi-square test or Fisher exact test (two-sided) was used to determine the correlations between parameters. Independent sample t-test (continuous variable) and chi-square/Fisher exact test (grouped variable) were conducted to determine the correlations between parameters. The results were considered significant when p-values were $<0.05$. Statistical analyses were conducted using the SPSS ver. 19.0 statistical software program (SPSS Inc., Chicago, IL, USA).

\section{RESULTS}

\section{Cohort characteristics}

The 113 cases of resected major and minor salivary gland neoplasms included 82 benign tumors and 31 malignant tumors. The benign tumors consisted of 48 pleomorphic adenomas (58.5\%), 27 Warthin tumors (32.9\%), three basal cell adenomas
(3.7\%), three cystadenomas (3.7\%), and one oncocytoma (1.2\%). The malignant tumors consisted of six mucoepidermoid carcinomas, low grade (19.4\%); three mucoepidermoid carcinomas, intermediate grade $(9.7 \%)$; three mucoepidermoid carcinomas, high grade (9.7\%); four salivary duct carcinomas (12.9\%); three squamous cell carcinomas (9.7\%); three adenoid cystic carcinomas (9.7\%); two adenocarcinomas, NOS (6.5\%); and one each of carcinoma ex pleomorphic adenoma (CA ex-PA), polymorphous low-grade adenocarcinoma, epithelial myoepithelial carcinoma, myoepithelial carcinoma, acinic cell carcinoma, carcinosarcoma, and poorly differentiated carcinoma.

The clinical characteristics of all 113 cases are shown in Table 1. Benign tumors typically occurred in major salivary glands (81 of 82 cases, $98.8 \%$ ) whereas malignant tumors occurred in major salivary glands (18 of 31 cases, $58.1 \%$ ) and minor salivary glands $(13,41.9 \%)$. The tumor size was not significantly different between malignant and benign cases. The tumor lesion was surgically completely resected in all 82 benign cases and 26 of 31 malignant cases $(83.9 \%)$.

\section{PLAG1 expression is specific for pleomorphic adenoma}

Immunohistochemical staining revealed that PLAG1 protein was expressed in the nucleus (Fig. 1B). Cells in the normal salivary glands did not express PLAG1 (Fig. 1A). Among 113 cases, all 34 benign salivary gland neoplasms other than pleomorphic adenoma were PLAG1-negative. Additionally, all 31 malignant salivary gland neoplasms including one $\mathrm{Ca}$ ex-PA case were PLAG1-negative (Fig. 1C).

Among the 48 cases of pleomorphic adenoma, PLAG1 protein expression was observed in 29 (60.4\%). Histologically, all of the tumors were composed of epithelial and myoepithelial/stromal components in a mixture of patterns. PLAG1 expression was moderately to strongly positive in myoepithelial/stromal components, regardless of its negativity and/or faint positivity in the epithelial component (Fig. 1B).

Table 1. Characteristics of 113 salivary gland neoplasms

\begin{tabular}{lccc}
\hline & Benign $(n=82)$ & Malignant $(n=31)$ & $p$-value \\
\hline Age $(y r)$ & $52 \pm 14$ & $56 \pm 16$ & .165 \\
Sex & & & \\
$\quad$ Male & $48(58.5)$ & $18(58.1)$ & .964 \\
$\quad$ Female & $34(41.5)$ & $13(41.9)$ & \\
Site & & & \\
$\quad$ Major & $81(98.8)$ & $18(58.1)$ & $<.001$ \\
$\quad$ Minor & $1(1.2)$ & $13(41.9)$ & .661 \\
Size $(\mathrm{cm})$ & $2.6 \pm 1.3$ & $2.6 \pm 1.4$ & \\
\hline
\end{tabular}

Values are presented as mean \pm standard deviation or number (\%). 

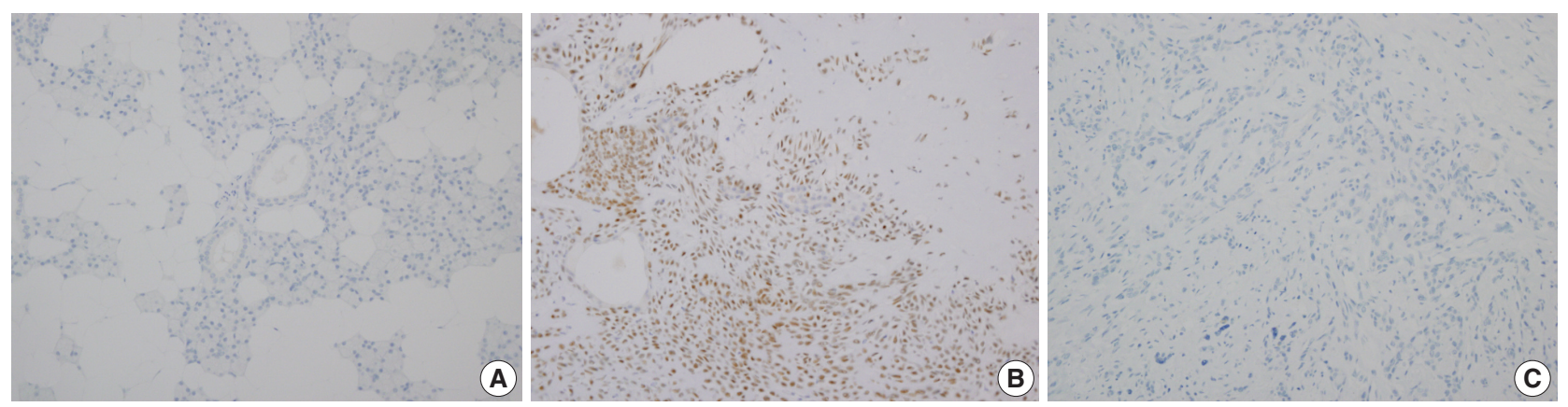

Fig. 1. Representative images of PLAG1 protein expression. (A) PLAG1 expression is not observed in the normal salivary gland parenchyme. (B) PLAG1 nuclear expression is observed in pleomorphic adenoma (myoepithelial cells). (C) PLAG1 expression is not observed in adenoid cystic carcinoma.

Table 2. Clinicopathological characteristics according to PLAG1 protein expression status in 48 pleomorphic adenoma cases

\begin{tabular}{lccc}
\hline & PLAG1-negative & PLAG1-positive & p-value \\
\hline Total & $19(39.6)$ & $29(60.4)$ & - \\
Age $(\mathrm{yr})$ & $48 \pm 12$ & $48 \pm 16$ & .985 \\
Sex & & & \\
$\quad$ Male & $8(40.0)$ & $12(60.0)$ & .960 \\
$\quad$ Female & $11(39.3)$ & $17(60.7)$ & \\
Size (cm) & $2.5 \pm 1.0$ & $2.6 \pm 1.4$ & .622 \\
Gland types & & & \\
$\quad$ Minor & 0 & $1(100)$ & 1.000 \\
$\quad$ Major & $19(40.4)$ & $28(59.6)$ & \\
Parotid & $18(48.6)$ & $19(51.4)$ & $.034^{\mathrm{a}}$ \\
Submandibular & $1(10.0)$ & $9(90.0)$ & \\
\hline
\end{tabular}

Values are presented as number (\%) or mean \pm standard deviation. Independent sample t-test (continuous variable) and chi-square/Fisher exact test (grouped variable) were conducted.

aStatistically significant.

PLAG1 positivity was not associated with age, sex, or tumor size and was higher in submandibular gland pleomorphic adenomas than in parotid gland pleomorphic adenomas $(90.0 \%$ vs $51.4 \%$, respectively); however, the difference was not significant (Table 2).

The sensitivity of PLAG1 expression by IHC was found to be $60.4 \%(45.3 \%-74.2 \%)$ and specificity was $100 \%(94.4 \%-$ $100 \%)$. The positive predictive value was $100 \%$ and negative predictive value was $77.1 \%(70.4 \%-82.7 \%)$.

\section{SOX10 expression indicates cell of origin}

SOX10 protein showed nuclear expression by IHC (Fig. 2). In normal salivary gland tissue, SOX10 staining was positive in intercalated ductal epithelial cells. Mucinous acinar cells were SOX10 negative, whereas serous acinar cells were SOX10 positive (Fig. 2A). The intensity of SOX10 nuclear staining was higher in salivary glands with atrophic change, and the intensity of staining in serous acinar cells was lower than that in interca- lated ductal epithelial cells.

For the 82 benign salivary gland neoplasms, all 48 pleomorphic adenoma cases and all three basal cell adenoma cases were SOX10positive. One pleomorphic adenoma showed scant positivity in most (>50\%) of the tumor cells, and we reclassified this case as SOX10-positive. All 27 Warthin tumors, one oncocytoma, and three cystadenoma cases were SOX10 negative. Although four cases of Warthin tumors also contained weakly positive tumor cells, the low proportion of positive cells $(<5 \%)$ was insufficient for reclassification.

Among the 31 malignant salivary gland neoplasms, nine (29.0\%) tumors were SOX10-positive including three of three adenoid cystic carcinoma, one of one CA ex-PA, one of one epithelial-myoepithelial carcinoma, one of one myoepithelial carcinoma, one of one poorly differentiated carcinoma, and one of one polymorphous low-grade adenocarcinoma. Scant but diffuse positivity was observed in one acinic cell carcinoma, and we added this case to the SOX10-positive group. All 12 cases of mucoepidermoid carcinoma and one carcinosarcoma were SOX10negative. Detailed results are provided in Table 3.

\section{Myb expression is relatively common in salivary gland neoplasms}

Myb expression showed nuclear and cytoplasmic staining, and the presence of nuclear staining was regarded as positive. Myb expression was not observed in the normal salivary gland parenchyme (Fig. 3A). Nuclear Myb expression was restricted to adenoid cystic carcinoma cells of myoepithelial origin (Fig. 3B).

Among the 82 benign salivary gland neoplasms, 17 cases (20.7\%) were Myb-positive. Specifically, 16 of 48 pleomorphic adenomas (33.3\%) and one of three basal cell adenomas (33.3\%) were Myb-positive. In contrast, all 27 Warthin tumors, one oncocytoma, and three cystadenomas showed Myb negativity.

For the 31 malignant tumors with available tissue microarray, 

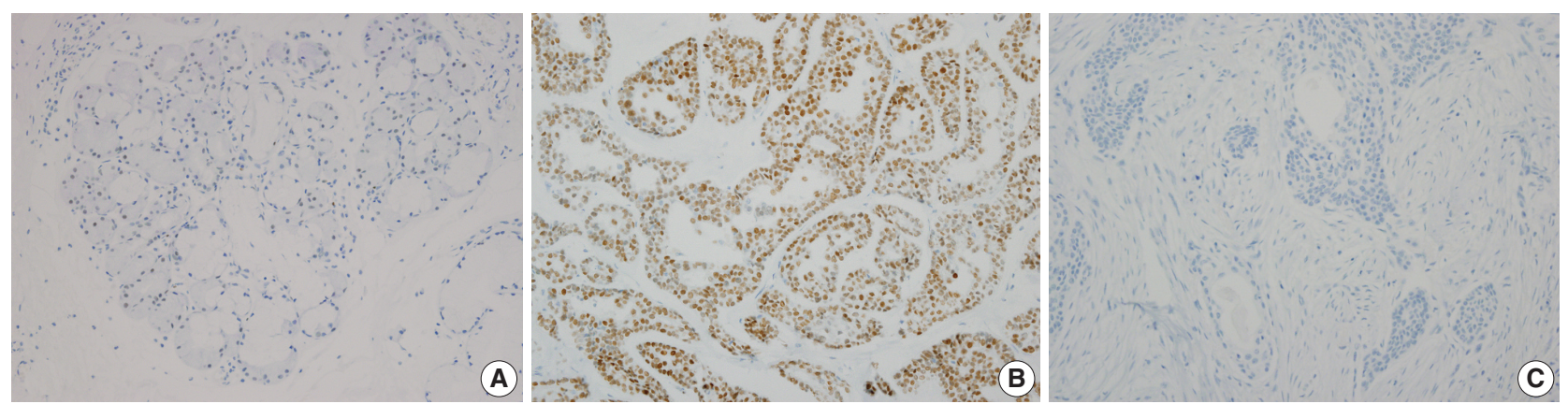

Fig. 2. Representative images of SOX10 protein expression. (A) SOX10 staining is not found in mucinous acinar cells. (B) SOX10 positivity is observed in adenoid cystic carcinoma. (C) SOX10 staining is not observed in mucoepidermoid carcinoma, low grade.

Table 3. SOX10 expression in malignant salivary gland neoplasms with different histologic types

\begin{tabular}{|c|c|c|}
\hline SOX10-positive $(n=9)$ & SOX10-negative $(n=22)$ & Correlative normal histology \\
\hline Acinic cell carcinoma $(n=1)$ & - & Acinus \\
\hline Adenoid cystic carcinoma $(n=3)$ & - & Intercalated duct \\
\hline Carcinoma ex pleomorphic adenoma $(n=1)$ & - & Intercalated duct \\
\hline Epithelial-myoepithelial carcinoma $(n=1)$ & - & Intercalated duct \\
\hline Myoepithelial carcinoma $(n=1)$ & - & Intercalated duct \\
\hline \multirow[t]{6}{*}{ Polymorphous low grade adenocarcinoma $(n=1)$} & - & Intercalated duct \\
\hline & Mucoepidermoid carcinoma, high grade $(n=3)$ & Excretory duct \\
\hline & Mucoepidermoid carcinoma, intermediate grade $(n=3)$ & Excretory duct \\
\hline & Mucoepidermoid carcinoma, low grade $(n=6)$ & Excretory duct \\
\hline & Salivary duct carcinoma $(n=4)$ & Excretory duct \\
\hline & Adenocarcinoma, NOS $(n=2)$ & NA \\
\hline \multirow[t]{3}{*}{ Poorly differentiated carcinoma $(n=1)$} & - & NA \\
\hline & Carcinosarcoma $(n=1)$ & NA \\
\hline & Squamous cell carcinoma $(n=3)$ & NA \\
\hline
\end{tabular}

NOS, not otherwise specified; NA, not applicable.
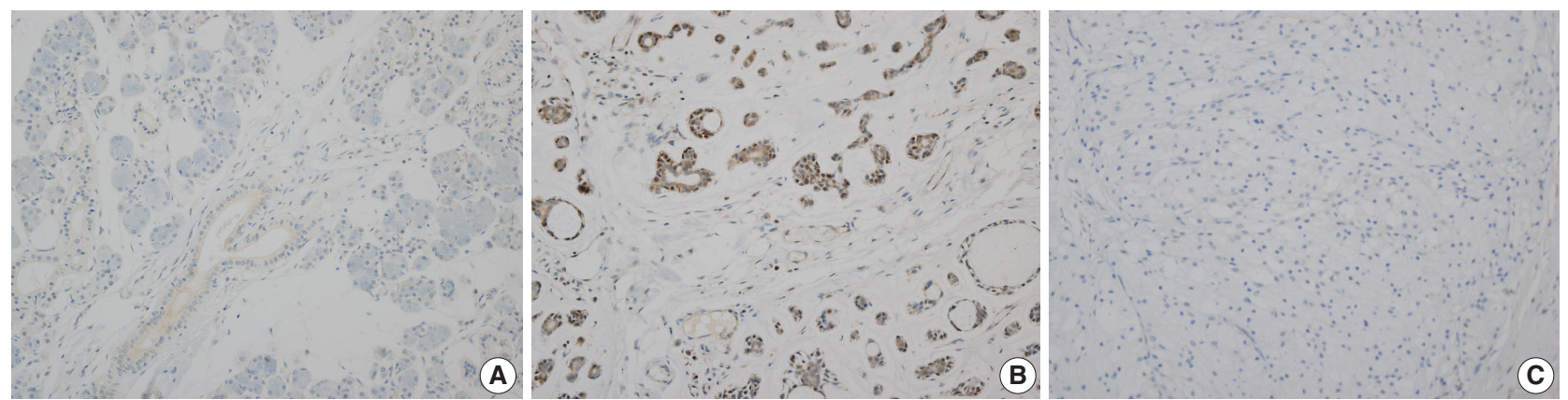

Fig. 3. Representative images of Myb protein expression. (A) Myb expression is not observed in the normal salivary gland parenchyme. (B) Myb positivity is observed in adenoid cystic carcinoma. (C) Myb protein is not observed in acinic cell carcinoma.

five tumors showed Myb positivity including all three adenoid cystic carcinomas, one myoepithelial carcinoma, and one salivary duct carcinoma. Detailed results are provided in Table 4. All 12 cases of mucoepidermoid carcinomas, one acinic cell carcinoma, one CA ex-PA, one carcinosarcoma, one epithelial-myoepithelial carcinoma, one poorly differentiated carcinoma, and one polymorphous low grade showed Myb negativity.
One representative paraffin tumor block ('whole section') was tested for Myb-positive cases. In three adenoid cystic carcinoma cases and one myoepithelial carcinoma case, moderate-to-strong nuclear staining of Myb was observed in a peripheral pattern. One salivary duct carcinoma case did not show expression of Myb in whole section staining. Three additional adenoid cystic carcinoma specimens that were resected recently (less than one 
Table 4. Immunohistochemical expression pattern of Myb-positive malignant salivary gland neoplasms

\begin{tabular}{|c|c|c|c|c|c|}
\hline \multirow{2}{*}{ Case No. } & \multirow{2}{*}{ Initial diagnosis } & \multicolumn{3}{|c|}{ Tissue microarray } & \multirow{2}{*}{ Final diagnosis } \\
\hline & & Nucleus & Cytoplasm & No. of positive cores & \\
\hline 37 & Adenoid cystic carcinoma (AdCC) & $2+/ 3$ & 0 & 3 of 3 & $\mathrm{AdCC}$ \\
\hline 15 & Adenoid cystic carcinoma & 0 & 0 & 0 of $3^{a}$ & AdCC \\
\hline 72 & Adenocarcinoma, NOS & $3+/ 3$ & $1+/ 3$ & 2 of 3 & AdCC \\
\hline 44 & Myoepithelial carcinoma & $3+/ 3$ & $1+/ 3$ & 3 of 3 & Myoepithelial carcinoma with AdCC-like feature \\
\hline 27 & Salivary duct carcinoma (SDC) & $1+/ 3$ & $1+/ 3$ & 1 of 3 & SDC \\
\hline
\end{tabular}

NOS, not otherwise specified.

${ }^{a}$ Myb positivity was observed in additional whole section staining.

year ago) were immunohistochemically tested and all three cases showed Myb positivity with a peripheral pattern.

\section{Relationships between markers}

SOX10 expression and Myb expression were significantly correlated ( $\mathrm{p}<.001)$. Only one case was Myb positive despite being SOX10 negative in tissue microarray, but this case was reclassified as Myb-negative after additional whole section staining. Finally, all Myb-positive cases showed SOX10 positivity.

In pleomorphic adenoma, 16 of 48 cases (33.3\%) showed Myb positivity. This trend was the same regardless of PLAG1 positivity or negativity (31.0\% and $36.8 \%$, respectively).

\section{DISCUSSION}

Salivary gland neoplasms exhibit extensive morphologic diversity and overlapping characteristics. Until recently, the classification of salivary gland tumors largely depended on histomorphological findings and cellular lineages. ${ }^{9}$ Recent advances have been made in salivary gland tumor pathology, particularly with the development of methods for analyzing molecular tumorigenesis. Previous studies showed that many subtypes of salivary gland tumors can be defined by tumor-specific translocation. ${ }^{10}$

Specific genetic rearrangements have been recognized as useful diagnostic markers of salivary gland neoplasms. Most secretory carcinomas harbor a $\mathrm{t}(12 ; 15)(\mathrm{p} 13 ; \mathrm{q} 25)$ translocation that results in ETV6-NTRK3 fusion': EWSR1-ATF1 fusion was detected in nearly all cases of hyalinizing clear cell carcinoma, and approximately $80 \%$ of low-grade mucoepidermoid carcinomas exhibited CRTC1-MAML2 fusion. ${ }^{2}$ MYB-NFIB fusion was reported in 28\%-86\% of adenoid cystic carcinoma cases, although the proto-oncogene $M Y B$ could also have other fusion partners. Fusion involving the PLAG1 locus has been reported in 24\%$88 \%$ of pleomorphic adenomas and CA ex-PA. ${ }^{11}$

Fluorescence in situ hybridization (FISH) is the gold-standard method for evaluating chromosomal rearrangements. However, this detection method is costly, requires a fluorescence microscope, and is time- and labor-intensive. ${ }^{12}$ Immunohistochemical staining is a reasonable and more economical alternative. IHC analysis is particularly advantageous in cases where oncoprotein overexpression results from low-level amplification or alternative mechanisms such as epigenetic pathways.

Several of the recognized cytogenetic alterations in pleomorphic adenoma are translocations involving 8q12 or 12q13-15.1 Researchers discovered that the oncogenes PLAG1 (located at 8q12) and HMGA2 (located at 12q15) are present in those particular lesions. ${ }^{9}$ In 2005, Martins et al. ${ }^{13}$ reported that PLAG1 gene alterations were observed in 14 of 16 cases (87.5\%) of salivary gland pleomorphic adenoma using in situ hybridization. Since then, five studies have reported PLAG1 immunopositivity in salivary pleomorphic adenoma, with a rate of $92 \%-100 \%$ positivity. ${ }^{11,14-17}$ Katabi et al. ${ }^{11}$ compared PLAG1 FISH and IHC results in salivary pleomorphic adenoma with positivity rates of 33\% (5/15) and 96\% (22/23), respectively. Matsuyama et $a l .{ }^{17}$ reported the proportion of PLAG1 gene alteration in salivary pleomorphic adenoma as $24 \%$ (11/45) using reverse transcription polymerase chain reaction whereas PLAG1 immunoreactivity was 100\% (45/45). This discrepancy could be explained by the existence of another mechanism for PLAG1 protein expression other than PLAG1 rearrangement. Matsuyama et al..$^{17}$ reported that some PLAG1-immunopositive PA cases did not exhibit PLAG1 rearrangements or express the HMGA2WIF1 fusion transcript. Moreover, promoter swapping mechanisms or low-level amplification of chromosome 8 result in PLAG1 overexpression in lipoblastoma. ${ }^{18}$ In human development, HMGA2 acts as a regulator of PLAG1 expression in the HMGA2-PLAG1-IGF2 pathway. ${ }^{19}$ Overexpression of PLAG1 was also reported in several other human tumors, such as lipoblastoma, hepatoblastoma, and acute myeloid leukemia, ${ }^{20}$ in the absence of consistent genetic fusion including PLAG1. Hence, PLAG1 fusion accounts for only a portion of PLAG1 protein overexpression in salivary gland PA. 
Our cohort included one case of CA ex-PA, which showed PLAG1 negativity. Whole-section staining was additionally conducted for the CA ex-PA case; however, PLAG1 immunoreactivity was not observed in the carcinoma or adenoma portions (not shown). Previous studies have attempted to distinguish between CA ex-PA and other carcinomas of the salivary glands using PLAG1 IHC. The proportion of PLAG1-immunopositive salivary CA ex-PA in studies including more than $10 \mathrm{CA}$ ex-PA cases was 35\%-60\%, which was lower than that of benign PA $(92 \%-100 \%)^{11,15,21}$

SOX10 IHC was recently used to diagnose Schwann cell and melanocytic tumors, particularly malignant peripheral nerve sheath tumor and malignant melanoma. ${ }^{5}$ Immunostaining using monoclonal anti-SOX10 antibody showed consistent results and therefore can be immediately applied in clinical diagnostic practice. Epithelial structures of normal salivary gland tissue are divided into four parts: acinus, intercalated duct, striated duct, and excretory duct. SOX10 was expressed in acinar cells, myoepithelial cells in acini, and luminal cells in intercalated ducts. ${ }^{6}$ Although each salivary gland tumor is thought to originate in the reserve cells and/or stem cell progenitors, histological similarities between the normal salivary gland tissue and the primary salivary gland neoplasms were widely observed. ${ }^{22}$ Pleomorphic adenoma, monomorphic adenoma (including basal cell adenoma), adenoid cystic carcinoma, epithelial-myoepithelial carcinoma, and polymorphous low-grade adenocarcinoma were considered to be related to the intercalated duct ${ }^{22}$ and all of them showed SOX10 positivity in our study (Table 3). In contrast, 27 Warthin tumors and one oncocytoma, which are considered to be of striated duct origin, and three cystadenomas, considered to be of excretory duct origin, showed SOX10 negativity. SOX10 appears to be a potential marker for cells of origin and differentiation in salivary gland tumors. ${ }^{23}$

In the literature, several authors have reported SOX10 positivity of acinic cell carcinoma (AciCC). ${ }^{6,23}$ Only one AciCC case was included in this study. Contrary to the findings of Hsieh $e t$ al., ${ }^{23}$ negative to scant positive tumor cells were observed in our case after whole section staining. This intensity was similar to the few positive cells found in four Warthin tumor cases. We concluded that our AciCC case was SOX10-positive, despite differing opinions among other authors. In our study, the staining intensity of SOX10 was low in normal serous acinus compared with intercalated ductal cells and intercalated duct-originated tumors, and mucinous acinar cells did not express SOX10 (Fig. 2A). Ohtomo et al. ${ }^{6}$ reported that SOX10 was weakly expressed in mucinous acinar cells and more intensely expressed in serous acinar cells. Considering this expression pattern in normal salivary glands, the scant positivity in our AciCC cases seems to be due to the difference in experimental conditions. Several factors may have contributed towards this low intensity including the facts that acinar cells contain digestive enzymes and that tissue of this case was collected 10 years before the study.

The avian myeloblastosis viral oncogene homolog v-Myb is a proto-oncogene located at $6 \mathrm{q} 22-23^{11}$ and the recurrent $\mathrm{t}(6 ; 9)$ (q22-23;p23-24) translocation is repeatedly observed in adenoid cystic carcinoma of the salivary glands. This translocation results in fusion of the MYB proto-oncogene with the transcription factor gene NFIB. ${ }^{7}$ Adenoid cystic carcinoma showed a unique histologic feature regardless of the organ of origin. Myb overexpression was detected in adenoid cystic carcinoma of the salivary glands, ${ }^{7,24}$ lacrimal glands, ${ }^{25,26}$ skin, ${ }^{27}$ and breast. ${ }^{28}$ The proportion of $M Y B$ translocation in salivary adenoid cystic carcinomas varied between studies, with values ranging from $28 \%$ to $86 \%$. In previous studies $65 \%$ to $82 \%$ of adenoid cystic carcinoma cases showed Myb protein expression. ${ }^{7,24}$ Mitani et al. ${ }^{24}$ conducted concurrent MYB-NFIB fusion FISH and Myb IHC and found that $61 \%$ (25 of 41) of adenoid cystic carcinomas without $M Y B-N F I B$ fusion showed Myb immunopositivity. In breast cancer, Myb IHC is more sensitive than MYB-NFIB FISH in the diagnosis of adenoid cystic carcinoma. ${ }^{28}$ In our study, one case showed Myb negativity according to the tissue microarray but focal Myb positivity of the peripheral pattern was observed in whole section staining. Brill et al.? also reported this phenomenon as a "peculiar zonal staining pattern." Since normal Myb has a half-life of approximately 30 minutes, ${ }^{29}$ the peripheral staining problem might be related to degradation and the fixation procedures used in daily practice. We also added three recent adenoid cystic carcinoma cases and all showed Myb positivity with the same staining pattern (not shown).

One case (No. 72) had ambiguous histologic features showing characteristics of both adenoid cystic carcinoma (AdCC) and adenocarcinoma, NOS at the time of primary diagnosis. After histologic review and consideration of Myb positivity, the authors concluded that this case was AdCC. Another Myb-positive case (No. 44) was initially diagnosed as myoepithelial carcinoma, and the tumor had foci of AdCC-like histologic features. Results of Myb IHC showed a marginal staining pattern and heterogeneous expression. Interpretation of Myb IHC requires caution when the study material is a biopsy or tissue microarray specimen; however, Myb IHC staining of the surgical specimen may be helpful when the diagnosis of AdCC is uncertain on histology.

In this study, we used newly developed IHC markers for the 
diagnostic evaluation of salivary gland neoplasms in a relatively large number of cases. We found that PLAG1 expression was specific to pleomorphic adenoma. SOX10 IHC may be helpful for diagnosing salivary gland neoplasms, which show acinus and/ or intercalated duct differentiation. Since SOX10 is expressed in various malignant tumors of salivary gland, its diagnostic value as a marker is relatively low. Myb immunohistochemistry is sensitive for adenoid cystic carcinoma detection in large resected specimens, especially when the tumor has ambiguous histology; however, caution is required for small biopsy specimens. Salivary gland tumors include a large and diverse group of tumors with overlapping histological features. New immunohistochemistry markers may play an important role in confirming the diagnosis of specific salivary gland tumors and broadening our understanding of salivary gland tumorigenesis.

\section{ORCID}

Ji Hyun Lee: https://orcid.org/0000-0002-7971-6516

Hye Ju Kang: https://orcid.org/0000-0002-2178-0005

Chong Woo Yoo: https://orcid.org/0000-0002-5221-4516

Weon Seo Park: https://orcid.org/0000-0002-0035-1455

Junsun Ryu: https://orcid.org/0000-0002-6730-6755

Yuh-Seog Jung: https://orcid.org/0000-0002-9467-4916

Sung Weon Choi: https://orcid.org/0000-0002-2038-2881

Joo Yong Park: https://orcid.org/0000-0002-0969-151X

Nayoung Han: https://orcid.org/0000-0001-8710-4280

\section{Conflicts of Interest}

The authors declare that they have no potential conflicts of interest.

\section{Acknowledgments}

This research was supported by a National Cancer Center of the Republic of Korea Grant (no. 1810022-1).

\section{REFERENCES}

1. Rosai J. Rosai and Ackerman's surgical pathology. Philadelphia: Mosby, 2011.

2. Griffith CC, Schmitt AC, Little JL, Magliocca KR. New Developments in salivary gland pathology: clinically useful ancillary testing and new potentially targetable molecular alterations. Arch Pathol Lab Med 2017; 141: 381-95.

3. Van Dyck F, Declercq J, Braem CV, Van de Ven WJ. PLAG1, the prototype of the PLAG gene family: versatility in tumour develop- ment (review). Int J Oncol 2007; 30: 765-74.

4. Zatkova A, Rouillard JM, Hartmann W, et al. Amplification and overexpression of the IGF2 regulator PLAG1 in hepatoblastoma. Genes Chromosomes Cancer 2004; 39: 126-37.

5. Dabbs DJ. Diagnostic immunohistochemistry: theranostic and genomic applications. Philadelphia: Saunders, 2018.

6. Ohtomo R, Mori T, Shibata S, et al. SOX10 is a novel marker of acinus and intercalated duct differentiation in salivary gland tumors: a clue to the histogenesis for tumor diagnosis. Mod Pathol 2013; 26: 1041-50.

7. Brill LB 2nd, Kanner WA, Fehr A, et al. Analysis of MYB expression and MYB-NFIB gene fusions in adenoid cystic carcinoma and other salivary neoplasms. Mod Pathol 2011; 24: 1169-76.

8. Kim SH, Park B, Joo J, et al. Retrospective analysis of 25 immunohistochemical tissue markers for differentiating multilocular cystic renal neoplasm of low malignant potential and multicystic renal cell carcinoma. Histol Histopathol 2018; 33: 589-96.

9. El-Naggar AK, Chan JK, Grandis JR, Takata T, Slootweg PJ. WHO classification of head and neck tumors. Lyon: IARC Press, 2017.

10. Vasef M, Auerbach A. Diagnostic pathology: molecular oncology. Philadelphia: Elsevier, 2016.

11. Katabi N, Xu B, Jungbluth AA, et al. PLAG1 immunohistochemistry is a sensitive marker for pleomorphic adenoma: a comparative study with PLAG1 genetic abnormalities. Histopathology 2018; 72: 285-93.

12. Han N, Kim MA, Lee HS, Kim WH. Evaluation of fibroblast growth factor receptor 2 expression, heterogeneity and clinical significance in gastric cancer. Pathobiology 2015; 82: 269-79.

13. Martins C, Fonseca I, Roque L, et al. PLAG1 gene alterations in salivary gland pleomorphic adenoma and carcinoma ex-pleomorphic adenoma: a combined study using chromosome banding, in situ hybridization and immunocytochemistry. Mod Pathol 2005; 18: 1048-55.

14. Rotellini M, Palomba A, Baroni G, Franchi A. Diagnostic utility of PLAG1 immunohistochemical determination in salivary gland tumors. Appl Immunohistochem Mol Morphol 2014; 22: 390-4.

15. de Brito BS, Giovanelli N, Egal ES, et al. Loss of expression of Plag1 in malignant transformation from pleomorphic adenoma to carcinoma ex pleomorphic adenoma. Hum Pathol 2016; 57: 152-9.

16. Avadhani V, Cohen C, Siddiqui MT. PLAG1: an immunohistochemical marker with limited utility in separating pleomorphic adenoma from other basaloid salivary gland tumors. Acta Cytol 2016; 60: 240-5.

17. Matsuyama A, Hisaoka M, Nagao Y, Hashimoto H. Aberrant PLAG1 expression in pleomorphic adenomas of the salivary gland: a molecular genetic and immunohistochemical study. Virchows Arch 2011; 458: 583-92. 
18. Deen M, Ebrahim S, Schloff D, Mohamed AN. A novel PLAG1RAD51L1 gene fusion resulting from a $t(8 ; 14)(q 12 ; q 24)$ in a case of lipoblastoma. Cancer Genet 2013; 206: 233-7.

19. Abi Habib W, Brioude F, Edouard T, et al. Genetic disruption of the oncogenic HMGA2-PLAG1-IGF2 pathway causes fetal growth restriction. Genet Med 2018; 20: 250-8.

20. Bartlett JM, Shaaban A, Schmitt F. Molecular pathology: a practical guide for the surgical pathologist and cytopathologist. Cambridge: Cambridge University Press, 2015.

21. Bahrami A, Dalton JD, Shivakumar B, Krane JF. PLAG1 alteration in carcinoma ex pleomorphic adenoma: immunohistochemical and fluorescence in situ hybridization studies of 22 cases. Head Neck Pathol 2012; 6: 328-35.

22. Mills SE. Histology for pathologists. Philadelphia: Lippincott Williams \& Wilkins, 2012.

23. Hsieh MS, Lee YH, Chang YL. SOX10-positive salivary gland tumors: a growing list, including mammary analogue secretory carcinoma of the salivary gland, sialoblastoma, low-grade salivary duct carcinoma, basal cell adenoma/adenocarcinoma, and a subgroup of mucoepidermoid carcinoma. Hum Pathol 2016; 56: 134-42.
24. Mitani Y, Li J, Rao PH, et al. Comprehensive analysis of the MYBNFIB gene fusion in salivary adenoid cystic carcinoma: incidence, variability, and clinicopathologic significance. Clin Cancer Res 2010; 16: 4722-31.

25. von Holstein SL, Fehr A, Persson M, et al. Adenoid cystic carcinoma of the lacrimal gland: $M Y B$ gene activation, genomic imbalances, and clinical characteristics. Ophthalmology 2013; 120: 2130-8.

26. Chen TY, Keeney MG, Chintakuntlawar AV, et al. Adenoid cystic carcinoma of the lacrimal gland is frequently characterized by MYB rearrangement. Eye (Lond) 2017; 31: 720-5.

27. North JP, McCalmont TH, Fehr A, van Zante A, Stenman G, LeBoit PE. Detection of MYB alterations and other immunohistochemical markers in primary cutaneous adenoid cystic carcinoma. Am J Surg Pathol 2015; 39: 1347-56.

28. Poling JS, Yonescu R, Subhawong AP, et al. MYB labeling by immunohistochemistry is more sensitive and specific for breast adenoid cystic carcinoma than MYB labeling by FISH. Am J Surg Pathol 2017; 41: 973-9.

29. Ramsay RG, Gonda TJ. MYB function in normal and cancer cells. Nat Rev Cancer 2008; 8: 523-34. 